\title{
Natriuretic Peptides' Role in Determining the Chemotherapy-Induced Nephrotoxicity and Their Value in Follow-Up
}

\author{
Mehmet Ali Erkurt ${ }^{\mathrm{a}, \mathrm{d}}$, Ismet Aydogdu ${ }^{\mathrm{b}}$, Irfan Kuku ${ }^{\mathrm{a}}$, Emin Kaya ${ }^{\mathrm{a}}$, \\ Melda Comert ${ }^{\mathrm{a}}$, Yalcin Basaran ${ }^{\mathrm{c}}$
}

\begin{abstract}
Background: Chemotherapy-induced nephrotoxicity is an important handicap for optimal treatment. For this reason, we need useful markers for early detection of chemotherapy-induced renal disfunction. This study was performed to investigate the relationship between the plasma natriuretic peptides' (ANP and BNP) levels and chemotherapy-induced nephrotoxicity.
\end{abstract}

Methods: Thirty patients treated with cisplatin, cyclophosphamide, doxorubicin and cytosine arabinoside which having known nephrotoxic side effects, were enrolled in this study. Seventeen of the patients were male and 13 were female with a median age of 44 . Also 30 healthy person were included to this study. Four chemotherapy courses were administered to each patient. Renal function tests (BUN, creatinin, urine micrototal protein/creatinin $[\mathrm{Pr} / \mathrm{Cr}]$, glomeruler filtration rate (GFR) and urine $\mathrm{Na}$ ) and plasma levels of ANP and BNP were measured before and after the treatments in both the patient and the control group.

Results: Before the treatment, there was no significant difference between the patients and the control group in comparison of renal function tests and plasma ANP-BNP levels. However, a decline in $\mathrm{GFR}$, increase in urine $\mathrm{Pr} / \mathrm{Cr}$ and plasma ANP-BNP levels were

Manuscript accepted for publication December 29, 2011

${ }^{a}$ Inonu University Faculty of Medicine, Department of Hematology, Malatya, Turkey

${ }^{b}$ Selcuk University Meram Faculty of Medicine, Department of Hematology, Konya, Turkey

${ }^{\mathrm{c}}$ Gulhane Military Medical Academy, Department of Internal Medicine, Ankara, Turkey

${ }^{\mathrm{d}}$ Corresponding author: Mehmet Ali Erkurt, Department of Hematology, School of Medicine, Turgut Ozal Medical Center, Inonu University, TR-44069 Malatya, Turkey. Email: erkurtali@hotmail.com

doi:10.4021/wjnu7e observed with subsequent courses of chemotherapy protocol, which were considered statistically significant $(\mathrm{P}<0.001)$. The plasma levels of ANP and BNP appeared to be higher in patients treated with nephrotoxic anticancer agents.

Conclusions: The elevated levels of natriuretic peptides may be useful in determining the chemotherapy-induced nephrotoxicity earlier, which highlights their importance and role in follow-up.

Keywords: Anticancer agents; Chemotherapy; Natriuretic peptide; Nephrotoxicity

\section{Introduction}

Chemotherapy-induced nephrotoxicity is an important obstacle for optimal treatment application in cancer patients. Several antineoplastic drugs may cause nephrotoxic effects which range from disorders of fluid and electrolyte balance, decreasing in GFR and permanent renal failure [1]. Earlier detection of renal dysfunction can prevent from chemotherapy-induced nephrotoxicity. For this reason, there are need useful biomarkers.

Natriuretic peptides consist a family of nine peptide hormones. Two of these peptides, ANP and BNP, are released from myocardial cells in the atrium and ventricles as a response to intravascular volume expansion and they have natriuretic and hypotensive effects [2]. They lower the resistance at the afferent arteriole and enhance the resistance at the efferent arteriole in the glomerulus. This results in augmented capillary hydraulic pressure which contributes to the increase in GFR and filtration fraction $[3,4]$. It has previously been shown that the circulating concentrations of natriuretic peptides are higher in patients with chronic renal failure and in hemodialysis patients, which appear to reflect the volume status $[5,6]$. Hemodialysis decreases the circulating concentrations of natriuretic peptides to be related with the volume status of the patients [7].

The nephrotoxic potential of cisplatin, cyclophosphamide, doxorubicin and high dose of cytosine arabinoside is known [1]. This current study was designed to investigate 
the association of natriuretic peptides with the nephrotoxicity of these antineoplastic drugs, and to introduce the role of ANP and BNP in predicting the nephrotoxicity.

\section{Materials and Methods}

Thirty patients who have treated with nephrotoxic antineoplastic drugs the Department of Hematology, Inonu University Faculty of Medicine, were enrolled in this study. Approval from the ethical review committee and ensuring informed consent from the patients and the control group, were obtained at the beginning of the study. Thirtytwo patients were enrolled in the study, but two of them were excluded due to chemotherapy related cardiac dysfunction during subsequent courses. Seventeen of the patients were male (57\%) and 13 of them were female (43\%). Their median age was 44 years (min: 20; $\max : 79)$. Twentysix of the patients were newly diagnosed and four of them were previously treated. The patients who were treated with drugs having proven nephrotoxic potency such as cisplatin, cyclophosphamide, doxorubicin and high dose cytosine arabinoside included in the study. Patients with a history of vascular disease, systemic hypertension, pulmonary hypertension, congestive heart failure, renal failure, hepatic cirrhosis, atrial fibrillation and tachycardia were excluded from the study. Patients with clinical findings such as edema, polyuria, and oliguria prior to the chemotherapy were also excluded. Serial echocardiographic evaluations before each course of chemotherapy were performed. Patients with left ventricular hypertrophy or cardiac dysfunction were excluded from the study. Fifteen healthy males and 15 healthy females who previously have not been treated with chemotherapeutic agents and who did not have renal dysfunction served as the control group. The control group also underwent an echocardiographic evaluation before the study and only healthy subjects served as control group. Their median age was 32 (min: 19; max: 66). Renal function tests and circulating ANP and BNP levels in both the patient and the control group were investigated before the treatment. Four courses of chemotherapy were given to each patient and after the treatment these tests were repeated (Table 1).

The GFR was estimated from the creatinine clearance. The creatinine clearance from a 24-hour urine collection was calculated using the following formula. Creatinine Clearance $(\mathrm{mL} / \mathrm{min})=$ Urine Creatinine $(\mathrm{mg} / \mathrm{dL}) \times$ Urine Volume $(\mathrm{mL} /$ day) / Plasma Creatinine $(\mathrm{mg} / \mathrm{dL}) \times 1440$ [8]. The normal value for GFR was accepted as $128 \pm 26 \mathrm{~mL} / \mathrm{min} / 1.73$ $\mathrm{m}^{2}$ for males, and $118 \pm 24 \mathrm{~mL} / \mathrm{min} / 1.73 \mathrm{~m}^{2}$ for females [9].

The proteinuria on the spot urine specimen was measured by nephelometric method using Dade Boehring BN-2 analyzer. The reference range of $\mathrm{Pr} / \mathrm{Cr}$ ratio on a spot urine specimen was determined to be below $200 \mathrm{mg} / \mathrm{dL}$ [10].

Blood urea nitrogen, creatinine, sodium, potassium, chloride, calcium, phosphate, magnesium, urine creatinine and urine sodium levels were examined by colorimetric method using Olympus 2700 analyzer. Blood and early morning spot urine samples were collected at 8:00 a.m. and the measurements were performed at the Biochemistry Laboratory of Inonu University Faculty of Medicine.

An expert who was not informed of the clinical and laboratory characteristics of the patients performed the laboratory assessment of the natriuretic peptides. Each patient was hydrated the night before the chemotherapy with approximately 3 liters of intravenous fluid. Blood samples were taken prior to each course before the initiation of volume administration and further blood samples were drawn 48 hours later following the chemotherapy. Blood samples were collected at 08:00 am in the defined days and the measurements were performed. They were collected through brachial vein to chilled plastic tubes containing aprotinin and ethylenediaminetetraacetic acid by a 19 sized needle. Then immediately placed on ice and promptly centrifuged during 15 minutes at $3000 \mathrm{rpm}\left(+4{ }^{\circ} \mathrm{C}\right)$. All samples were frozen and stored at $-80^{\circ} \mathrm{C}$ for following analysis. Plasma samples were defrosted on the day after the fourth course and analyzed for the concentrations of natriuretic peptides. ANP and BNP was detected using fluorescence immunoassay (Biotek Diagnostics, CA; Reader: ELx800; Kit: Phoenix Pharmaceuticals Inc Harbor Boulevard, Belmond, California 94002). This test was at the quality of determining the circulating levels of ANP and BNP at $0.1 \mathrm{pg} / \mathrm{mL}-100 \mathrm{pg} / \mathrm{mL}$ sensitivity. Prior to measurement, the plasma samples were raised to room temperature to become homogeneous and hemolytic samples were not used. A reaction was achieved principally by using murine ANP and BNP antibodies after transferring $50 \mu \mathrm{l}$ of sample and polyclonal antibodies were labeled with fluorescent dye. Test apparatus was containing two internal controls and two external liquid controls, and QC simulator was used by the purpose of control.

\section{Statistical methods}

Statistical analysis was performed using Statistical Package for Social Sciences (SPSS) Chicago USA for Windows 13.0 programme. Parametric data were expressed as mean \pm standard deviation and non-parametric data as percent. Comparisons between quantitative data of different courses in patient group were made by using a one-way ANOVA with Tukey's pairwise comparison procedure to control for multiple testing. The determinants of natriuretic peptide levels were investigated by multivariate regression analysis. Differences were considered statistically significant if $\mathrm{P}<0.05$.

\section{Results}

The patients were compared with each other according to 


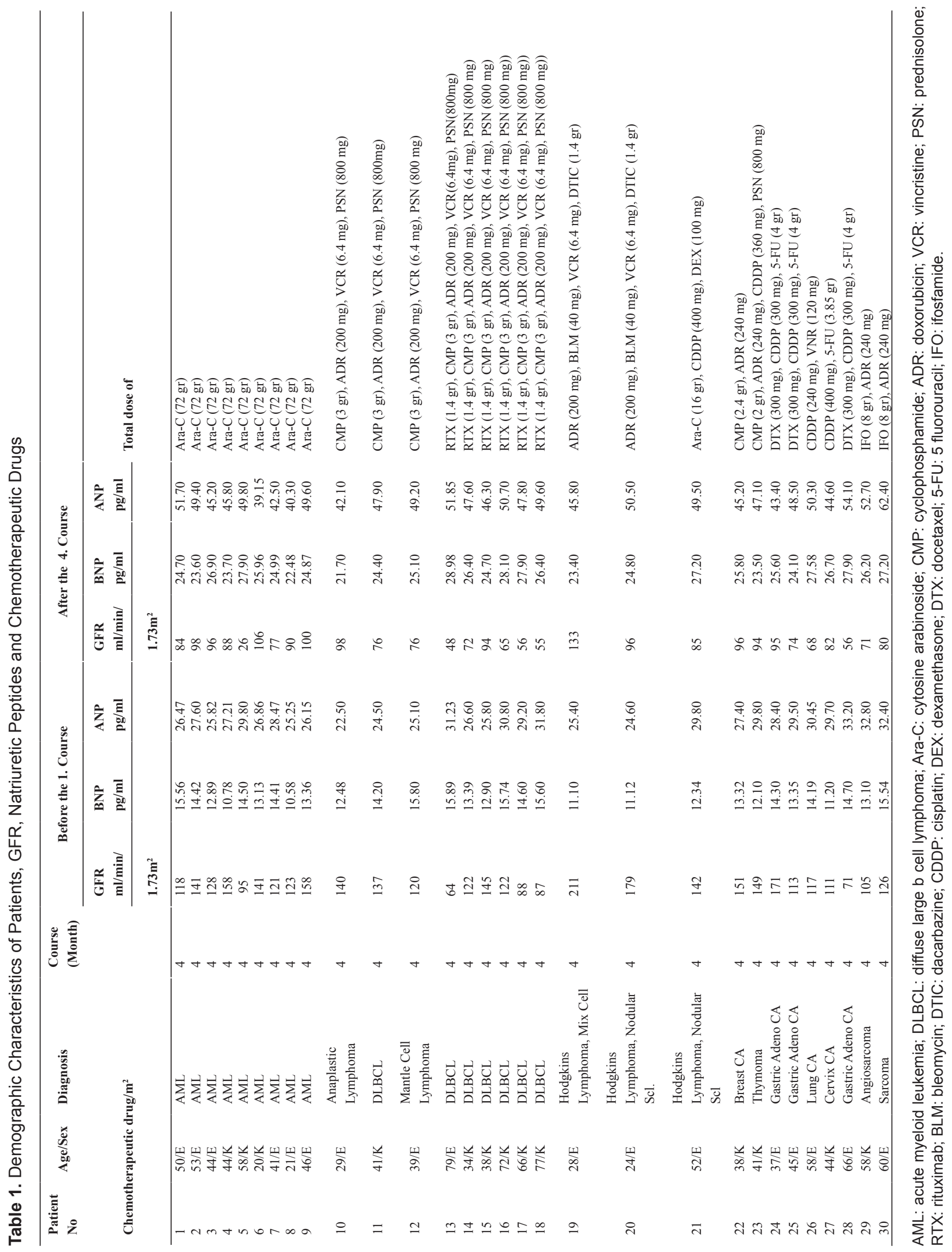




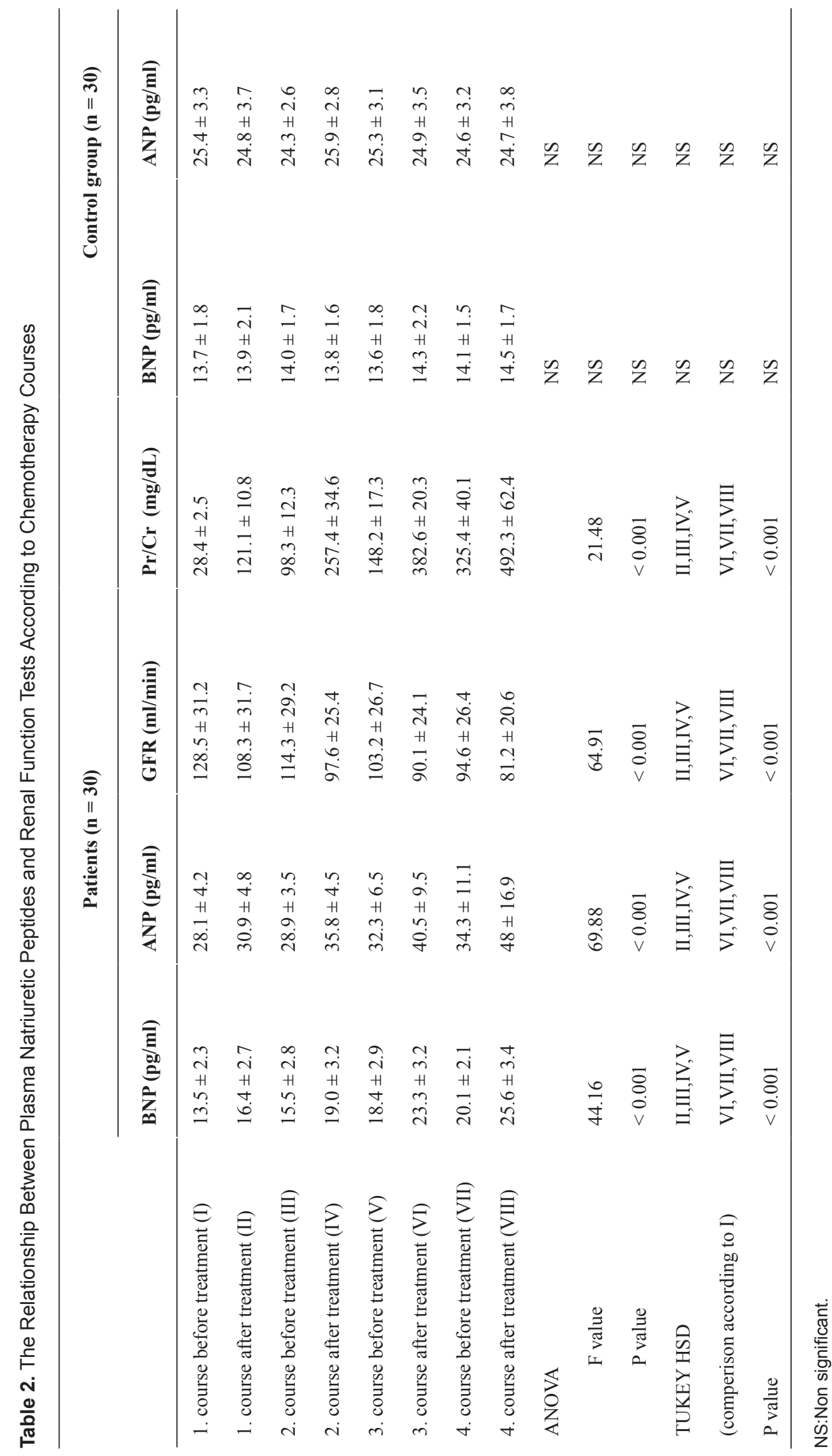


chemotherapy courses. Before the first course, mean GFR in the patient group was $128 \mathrm{~mL} / \mathrm{min}$ and $130 \mathrm{~mL} / \mathrm{min}$ in the control group. There was no significant difference between the groups $(P=0.17)$. Mean GFR significantly decreased with subsequent courses. The values of GFR before the first course, during the treatment and after the fourth course (F: 64.91 $\mathrm{P}<0.001$ ) indicated in Table 2.

In a patient (patient no 5, 3.3\%) although the GFR before the first course was within normal levels $(95 \mathrm{~mL} / \mathrm{min})$, renal disfunction progressed $(26 \mathrm{~mL} / \mathrm{min})$ after the fourth course. Dialysis was not required. After the fourth course, reduction in GFR was detected in other patients but no uremic complications were observed. Mean GFR values returned to normal spontaneously and gradually within two months.

Before the first course, mean $\mathrm{Pr} / \mathrm{Cr}$ ratio in the patient group was $28.4 \mathrm{mg} / \mathrm{dL}$, while it was $26.2 \mathrm{mg} / \mathrm{dL}$ in the control group. There was no significant difference between the groups $(\mathrm{P}=0.12)$. Mean $\mathrm{Pr} / \mathrm{Cr}$ ratio significantly increased with subsequent courses. Mean $\mathrm{Pr} / \mathrm{Cr}$ ratio significantly increased in parallel with a significant decline in mean GFR with subsequent courses (F: $21.48 \mathrm{P}<0.001)$ (Table 2). After the fourth course, the mean $\mathrm{Pr} / \mathrm{Cr}$ ratio returned to normal spontaneously within two months. After the third course, $\mathrm{Pr} /$ $\mathrm{Cr}$ ratio was exceed $1500 \mathrm{mg} /$ day in a patient (patient no $21,3.3 \%$ ). After supportive treatment it fall dawn to normal range.

Mean serum creatinine level before the first course was $0.69 \pm 0.11 \mathrm{mg} / \mathrm{dL}$ and $1.1 \pm 0.29 \mathrm{mg} / \mathrm{dL}$ after the fourth course, respectively. Mean serum creatinine levels after the treatment were determined significantly higher $(\mathrm{P}<0.001)$, when compared with the levels before the treatment.

Before the first course, mean ANP levels in the patient group was $28.1 \mathrm{pg} / \mathrm{mL}$, while it was $25.4 \mathrm{pg} / \mathrm{mL}$ in the control group. There was no significant difference between the groups $(P=0.19)$. After the fourth course, mean ANP levels in the patient group was $48 \mathrm{pg} / \mathrm{mL}$, while it was $24.7 \mathrm{pg} /$ $\mathrm{mL}$ in the control group. Mean ANP levels significantly increased with subsequent courses (F: 69.88, $\mathrm{P} \leq 0.001)$ (Table 2).

Before the first course, mean BNP levels in the patient group was $13.5 \mathrm{pg} / \mathrm{mL}$, while it was $13.8 \mathrm{pg} / \mathrm{mL}$ in the control group. There was no significant difference between the two groups $(\mathrm{P}=0.21)$. After the fourth course, mean BNP levels in the patient group was $25.6 \mathrm{pg} / \mathrm{mL}$, while it was 14.5 $\mathrm{pg} / \mathrm{mL}$ in the control group. Mean BNP levels significantly increased with subsequent courses (F: 44.16, P <0.001) (Table 2).

A marked decline in GFR was observed with subsequent courses. ANP, BNP levels and urine $\mathrm{Pr} / \mathrm{Cr}$ ratio progressively increased. Differences in variables with subsequent courses were considered statistically significant compared with the first course $(\mathrm{P}<0.001)$ (Table 2).

Levels of natriuretic peptides increased in parallel with aggravation of kidney function tests. Kidney function tests decreased to baseline levels gradually within two months after the completion of fourth chemotherapy. A permanent kidney dysfunction did not develop.

\section{Discussion}

Nephrotoxicity is one of the most important side effects of the chemotherapeutic agents. Antimetabolites, alkylating agents and anthracyclines are most commonly used antineoplastic drugs that can cause nephrotoxicity [11]. Nephrotoxicity is an adverse effect that can disrupt regularly administration of anticancer treatments. Chemotherapeutic agents affect different segments of the nephron, which is the basic unit of the kidney: especially the glomerulus, proximal and distal tubules [1]. In this study, chemotherapy-induced nephrotoxicity which including progressive reduction in GFR and concurrent elevation in serum creatinine and urine $\mathrm{Pr} / \mathrm{Cr}$ ratio was observed. In our study, gradual improvement of renal function tests of the patients were established within two months after completion of chemotherapy. Permanent renal dysfunction was not occured in any patient. This situation can be explained by relatively small size of our study group.

ANP and BNP are produced predominantly by atrial and ventricular cells generally in response to volume or pressure overload and they both exerts natriuretic and vasodilatory effects [2]. They decrease the resistance at the afferent arteriole; and increase the resistance at the efferent arteriole in the glomerulus. Thus, resulting in augmented capillary hydraulic pressure which contributes to the increase in GFR and filtration fraction [3, 4]. A number of studies have shown that the circulating concentrations of natriuretic peptides are higher in patients with congestive heart failure, essential hypertension, hepatic cirrhosis, atrial fibrillation and tachycardia which appear to reflect volume status $[12,13]$. The disease state associated with the highest intravascular overload is renal failure because of the reduction of GFR. Plasma levels of ANP and BNP are almost always raised in end stage renal disease patients undergoing hemodialysis and in the late predialysis stage $[5,7,14]$. Fluid removal by hemofiltration lowers the plasma ANP levels [7]. In addition to a reduction in the blood urea nitrogen and plasma creatinine concentration, a reduction in circulating ANP levels by $34-42$ percent may occur by hemodialysis. The number of natriuretic peptide clearance receptors decreases by the loss of the renal parenchymal mass in chronic kidney disease. The circulating concentrations of the natriuretic peptides normalizes within a week after the treatment of the end stage renal disease with renal transplantation.

Decreased renal parenchyma functions results with decreased degradation of ANP and BNP and higher circulating concentrations of these peptides than the other diseases associated with intravascular overload [14]. A significant increase in ANP and BNP levels after the treatment was observed in 
this study between both the same and the different courses of the chemotherapy, compared with the control group. This increase was negatively correlated with the reduction in GFR that was used clinically to assess the degree of kidney impairment and positively correlated with the rise in urine $\mathrm{Pr} / \mathrm{Cr}$ ratio. The most likely causes of the increase in natriuretic peptide levels in our patients receiving chemotherapy are decline in the GFR produced by chemotherapy-induced nephrotoxicity resulting in retention of water and sodium, and the volume expansion due to renal dysfunction, as mentioned above. Chemotherapy can potentiate renal dysfunction and contribute the nephrotoxic potential, thus further increase in circulating levels of natriuretic peptides is expected with subsequent courses. Monitoring plasma levels of ANP and BNP in patients receiving chemotherapy may reflect a chance of the nephrotoxicity to be detected earlier. Although impairment in renal functions was statistically significant, it was not severe enough to require discontinuation of the chemotherapy. We reported the results in patients treated with median 4 course regimens of chemotherapy. However, some types of cancer require administration more courses of chemotherapy. High dose chemotherapy or prolonged time may aggravate renal dysfunction resulting in discontinuation of the therapy. Throughout these results, it is not possible to determine a threshold plasma level of natriuretic peptides after each subsequent chemotherapy course for discontinuation of therapy or limitation of dose in follow-up of chemotherapyinduced nephrotoxicity. To draw such conclusion further prospective studies are needed in a large number of patients.

In many studies, the circulating levels of natriuretic peptides have been reported to be higher in patients with kidney disease. Among these studies, only Franz et al reported patients with impaired renal function to have a dramatic increase of circulating levels of ANP and BNP even before serum creatinine levels start to rise [15]. Currently, in the literature there is still not enough knowledgement about the relationship between the nephrotoxicity of patients treated with chemotherapeutic drugs and circulating levels of ANP and BNP. In this study, the circulating levels of ANP and BNP were found to be higher in antineoplastic nephrotoxic agents receiving patients. This study highlights the importance of natriuretic peptide levels in determining the chemotherapy-induced nephrotoxicity and their role in follow-up. They may be useful in considering some preventive strategies in the patients treated with antineoplastic drugs before the development of nephrotoxicity. In diary pratice, creatin level is used for detection of nephrotoxicity. However, creatin level is not a sufficient indicator in the patients with low muscle mass and elderly. Therefore, in these patients, elevated levels of natriuretic peptides may be useful in determining the chemotherapy-induced nephrotoxicity earlier, which highlights their importance and the role in follow-up.

In conclusions, the circulating concentrations of ANP and BNP appear to be suggestive indicators of the chemo- therapy-induced nephrotoxicity and may be useful in long term follow-up. However, further studies are required in a larger number of patients to confirm our findings.

\section{References}

1. Kintzel PE. Anticancer drug-induced kidney disorders. Drug Saf. 2001;24(1):19-38.

2. Vesely DL. Natriuretic peptides and acute renal failure. Am J Physiol Renal Physiol. 2003;285(2):F167-177.

3. Genest J, Cantin M. Atrial natriuretic factor. Circulation. 1987;75(1 Pt 2):I118-124.

4. Pai S, Cutler R. Atrial natriuretic peptide. A Minireview Part II. Dialysis Transplantation J 1988; 17: 262-264.

5. Albano J, Raman G. Atrial natriuretic peptide in adults on Hemodialysis. Nephrology Dialysis Transplantation 1986; 1: 128-129.

6. Saxenhofer H, Gnadinger MP, Weidmann P, Shaw S, Schohn D, Hess C, Uehlinger DE, et al. Plasma levels and dialysance of atrial natriuretic peptide in terminal renal failure. Kidney Int. 1987;32(4):554-561.

7. Zoccali C, Ciccarelli M, Mallamaci F, Delfino D, Salnitro F, Parlongo S, Maggiore Q. Effect of ultrafiltration on plasma concentrations of atrial natriuretic peptide in haemodialysis patients. Nephrol Dial Transplant. 1986;1(3):188-191.

8. Levin N, Eknoyan G, Pipp M, Steinberg E. National Kidney Foundation: Dialysis Outcome Quality Initiative--development of methodology for clinical practice guidelines. Nephrol Dial Transplant. 1997;12(10):20602063.

9. Davies DF, Shock NW. Age changes in glomerular filtration rate, effective renal plasma flow, and tubular excretory capacity in adult males. J Clin Invest. 1950;29(5):496-507.

10. Warram JH, Gearin G, Laffel L, Krolewski AS. Effect of duration of type I diabetes on the prevalence of stages of diabetic nephropathy defined by urinary albumin/creatinine ratio. J Am Soc Nephrol. 1996;7(6):930-937.

11. Ikarashi Y, Kakihara T, Imai C, Tanaka A, Watanabe A, Uchiyama M. Glomerular dysfunction, independent of tubular dysfunction, induced by antineoplastic chemotherapy in children. Pediatr Int. 2004;46(5):570-575.

12. Omland T, Bonarjee VV, Lie RT, Caidahl K. Neurohumoral measurements as indicators of long-term prognosis after acute myocardial infarction. Am J Cardiol. 1995;76(4):230-235.

13. Takami Y, Horio T, Iwashima Y, Takiuchi S, Kamide K, Yoshihara F, Nakamura S, et al. Diagnostic and prognostic value of plasma brain natriuretic peptide in non-dialysisdependent CRF. Am J Kidney Dis. 2004;44(3):420-428.

14. Winters CJ, Vesely DL. Change in plasma immunoreactive N-terminus, C-terminus, and 4,000-dalton midpor- 
tion of atrial natriuretic factor prohormone with hemodialysis. Nephron. 1991;58(1):17-22.

15. Franz M, Woloszczuk W, Horl WH. Plasma concentra- tion and urinary excretion of N-terminal proatrial natriuretic peptides in patients with kidney diseases. Kidney Int. 2001;59(5):1928-1934. 